\title{
Supplementary experimental procedure
}

\section{Two-dimensional gel electrophoresis (2-DE)}

Human fibroblasts were cultured and mitochondria were isolated as described in mitochondria purification and preparation of protein samples for 2-D DIGE. Mitochondrial proteins for 2-DE were solubilized in a lysis buffer containing $8.4 \mathrm{M}$ urea, 2.4 M thiourea, 5\% CHAPS, 2 mM TCEP-HCl, and 1\% carrier ampholytes $\mathrm{pH} 3-$ 10. Samples were agitated for 1 hour at room temperature, and cleaned with PlusOne 2DE Clean-up Kit (GE Healthcare). After two hours agitation at room temperature, samples were dialyzed against lysis buffer and protein content was determined using the RC DC Protein Assay (Bio-Rad). Approximately, $0.5 \mathrm{mg}$ was the amount of total mitochondrial protein obtained from five T150 flasks.

For first dimension, IPG strips were rehydrated in the Immobiline DryStrip Reswelling Tray for 10 or 12 hours. Rehydration solution contained 8.4 M urea, 2.4 M thiourea, 2\% CHAPS, $2 \mathrm{mM}$ TCEP-HCl, and 0.5\% carrier ampholytes (pH range as the immobiline drystrip to be rehydrated). When basic $\mathrm{pH}$ range strips were used, reductor reagent was omitted and hydroxyethyl disulfide (DeStreak ${ }^{\mathrm{TM}}$, GE Healthcare) was added to the rehydration solution to a final concentration of $100 \mathrm{mM}$. Mitochondrial proteins were diluted with rehydration buffer and applied directly to the rehydrated IPG strip through the sample cup (0.08 mg and $0.1 \mathrm{mg}$ total protein when IPG strips pH 3-10 and 6-11 were used, and $0.25 \mathrm{mg}$ total protein when IPG strip pH 5-6 was used). Protein separation was performed using $18 \mathrm{~cm}$ pH range IPGs (3-10, 6-11 and 5-6) from GE Healthcare. The strips were subjected to electrophoresis using an Ettan IPGphor Isoelectric Focusing system (GE Healthcare) according to the manufacturer's instructions. After isoelectric focusing separation, strips were equilibrated twice for 15 min with solutions containing $50 \mathrm{mM}$ Tris- $\mathrm{HCl} \mathrm{pH}$ 8.8, $6 \mathrm{M}$ urea, 30\% glycerol, 2\% 
SDS, and traces of bromophenol blue. Dithiothreitol (1\%) and iodoacetamide (4\%) were added in the solutions of the first and second equilibration steps, respectively. The second-dimensional separation was performed on 10\% SDS-polyacrylamide gels. Gels were run at $6 \mathrm{~mA}$ per gel for about 15 hours at $4^{\circ} \mathrm{C}$, and silver stained. Proteins were identified by MALDI-TOF as described in mass spectrometry section (materials and methods). 
Supplementary table A. Mitochondrial proteins from human fibroblasts identified by 2-DE/MS.

\begin{tabular}{|c|c|c|c|c|c|c|c|c|}
\hline $\begin{array}{l}\text { Accession } \\
\text { number }^{a}\end{array}$ & Protein name & Location $^{b}$ & $\mathrm{Mr} / \mathrm{p} I^{c}$ & $\begin{array}{c}\text { Number of } \\
\text { masses searched }\end{array}$ & $\begin{array}{c}\text { Number of } \\
\text { masses matched }\end{array}$ & $\begin{array}{c}\text { Sequence } \\
\text { coverage \% }\end{array}$ & $\begin{array}{c}\text { MASCOT } \\
\text { score }\end{array}$ & $\begin{array}{l}\text { No. } / \\
\text { Figure }\end{array}$ \\
\hline Q9BWK8 & $\begin{array}{l}\text { Voltage-dependent anion } \\
\text { channel } 2\end{array}$ & OMM & $30412 / 6.81$ & 17 & 11 & 42 & 144 & $2 / \mathrm{A}$ \\
\hline Q9P129 & Calcium binding transporter & OMM & $45819 / 5.31$ & 18 & 7 & 16 & 81 & $18 / \mathrm{C}$ \\
\hline O96008 & TOM40 & OMM & 37893/6.79 & 7 & 6 & 21 & 105 & $36 / \mathrm{B}$ \\
\hline Q8IXV2 & $\begin{array}{l}\text { ATP synthase A chain, } \\
\text { mitochondrial }\end{array}$ & MIM & $60785 / 9.14$ & 8 & 5 & 16 & 76 & $37 / \mathrm{B}$ \\
\hline P24539 & $\begin{array}{l}\text { ATP synthase B chain, } \\
\text { mitochondrial }\end{array}$ & MIM & 28894/9.37 & 7 & 7 & 32 & 143 & 38/B \\
\hline O75947 & $\begin{array}{l}\text { ATP synthase D chain, } \\
\text { mitochondrial }\end{array}$ & MIM & $15773 / 6.61$ & 14 & 8 & 62 & 150 & $1 / \mathrm{A}$ \\
\hline P31040 & $\begin{array}{l}\text { Succinate dehydrogenase } \\
\text { [ubiquinone] flavoprotein } \\
\text { subunit }\end{array}$ & MIM & 72691/7.00 & 22 & 11 & 18 & 122 & 4/A \\
\hline A48043 & Ubiquinol-cytochrome-C- & MIM & $52618 / 5.94$ & 21 & 15 & 38 & 224 & $19 / \mathrm{C}$ \\
\hline
\end{tabular}




\begin{tabular}{|c|c|c|c|c|c|c|c|c|}
\hline & reductase core protein I & & & & & & & \\
\hline P22695 & $\begin{array}{l}\text { Ubiquinol-cytochrome C } \\
\text { reductase core protein } 2\end{array}$ & MIM & $48470 / 8.74$ & 6 & 4 & 13 & 63 & 39/B \\
\hline O75489 & $\begin{array}{l}\text { NADH-ubiquinone } \\
\text { oxidoreductase } 30 \mathrm{kDa} \text { subunit }\end{array}$ & MIM & $30241 / 7.6$ & 20 & 9 & 38 & 133 & 20/C \\
\hline P19404 & $\begin{array}{l}\text { NADH-ubiquinone } \\
\text { oxidoreductase } 24 \mathrm{kDa} \text { subunit }\end{array}$ & MIM & 27391/8.22 & 9 & 6 & 33 & 107 & 21/C \\
\hline Q16891 & Mitofilin (motor protein) & MIM & $83677 / 6.00$ & 13 & 10 & 18 & 144 & $22 / \mathrm{C}$ \\
\hline P43304 & $\begin{array}{l}\text { Glycerol-3-phosphate } \\
\text { dehydrogenase }\end{array}$ & MIM & $80833 / 7.23$ & 10 & 8 & 12 & 110 & 8/A \\
\hline Q96FZ8 & Citrate synthase & MM & $51712 / 8.40$ & 20 & 10 & 26 & 107 & 3/A \\
\hline KIHUA3 & $\begin{array}{l}\text { Nucleoside-triphosphate- } \\
\text { adenylate kinase }\end{array}$ & MM & $25268 / 8.47$ & 10 & 6 & 27 & 103 & $5 / \mathrm{A}$ \\
\hline P26440 & Isovaleryl-CoA dehydrogenase & MM & 46319/8.45 & 12 & 11 & 26 & 120 & 6/A \\
\hline P00367 & $\begin{array}{l}\text { Glutamate dehydrogenase } 1 \text {, } \\
\text { mitochondrial }\end{array}$ & MM & $61397 / 7.66$ & 20 & 9 & 21 & 109 & 7/A \\
\hline Q02218 & 2-oxoglutarate dehydrogenase & MM & $113475 / 6.62$ & 31 & 17 & 17 & 154 & 9/A \\
\hline
\end{tabular}




\begin{tabular}{|c|c|c|c|c|c|c|c|c|}
\hline & E1 component, mitochondrial & & & & & & & \\
\hline P04179 & Superoxide dismutase & MM & $24722 / 8.35$ & 12 & 7 & 30 & 122 & $10 / \mathrm{A}$ \\
\hline P28330 & $\begin{array}{l}\text { Acyl-CoA dehydrogenase, } \\
\text { long-chain specific }\end{array}$ & MM & $47669 / 7.28$ & 31 & 11 & 21 & 106 & $11 / \mathrm{A}$ \\
\hline P13804 & $\begin{array}{l}\text { Electron transfer flavoprotein, } \\
\text { alpha subunit }\end{array}$ & MM & $35079 / 8.62$ & 8 & 7 & 25 & 128 & $12 / \mathrm{A}$ \\
\hline P15121 & Aldose reductase & $\mathrm{MM}$ & $35722 / 6.55$ & 12 & 8 & 24 & 86 & $13 / \mathrm{A}$ \\
\hline Q8N2B8 & $\begin{array}{l}\text { Heat shock 70kD protein 9B } \\
\text { (Mortalin-2) }\end{array}$ & MM & $73853 / 6.00$ & 7 & 6 & 15 & 91 & $14 / \mathrm{A}$ \\
\hline P50213 & $\begin{array}{l}\text { Isocitrate dehydrogenase } \alpha \text { - } \\
\text { chain }\end{array}$ & MM & $39591 / 6.46$ & 31 & 11 & 29 & 80 & $15 / \mathrm{A}$ \\
\hline Q96H54 & $\begin{array}{l}\text { Enoyl-CoA hydratase short } \\
\text { chain 1, mitochondrial }\end{array}$ & MM & 31399/8.34 & 22 & 7 & 39 & 81 & $16 / \mathrm{A}$ \\
\hline P30048 & $\begin{array}{l}\text { Thioredoxin-dependent } \\
\text { peroxide reductase } \\
\text { (Peroxiredoxin 3) }\end{array}$ & MM & $27692 / 7.68$ & 10 & 6 & 24 & 97 & $23 / \mathrm{C}$ \\
\hline P31937 & $\begin{array}{l}\text { 3-hydroxyisobutyrate } \\
\text { dehydrogenase }\end{array}$ & MM & $35329 / 8.10$ & 10 & 5 & 22 & 79 & $24 / \mathrm{C}$ \\
\hline
\end{tabular}




\begin{tabular}{|c|c|c|c|c|c|c|c|c|}
\hline AAH01633 & $\begin{array}{l}\text { Tu translation elongation } \\
\text { factor, mitochondrial }\end{array}$ & $\mathrm{MM}$ & $49541 / 7.26$ & 19 & 9 & 21 & 115 & $25 / C$ \\
\hline Q16822 & $\begin{array}{l}\text { Phosphoenolpyruvate } \\
\text { carboxykinase }\end{array}$ & MM & $70637 / 7.56$ & 14 & 7 & 12 & 80 & $26 / C$ \\
\hline P45954 & $\begin{array}{l}\text { Acyl Co-A dehydrogenase } \\
\text { short-chain-specific }\end{array}$ & $\mathrm{MM}$ & $47485 / 6.53$ & 6 & 5 & 13 & 82 & $27 / C$ \\
\hline Q9P2R7 & $\begin{array}{l}\text { ATP-specific succinyl-CoA } \\
\text { synthetase } \beta \text {-subunit }\end{array}$ & $\mathrm{MM}$ & $50331 / 7.00$ & 7 & 5 & 9 & 77 & $28 / C$ \\
\hline P11177 & $\begin{array}{l}\text { Pyruvate dehydrogenase E1 } \\
\text { component } \beta \text {-chain }\end{array}$ & MM & $39219 / 6.2$ & 10 & 6 & 20 & 92 & $29 / C$ \\
\hline P08559 & $\begin{array}{l}\text { Pyruvate dehydrogenase E1 } \\
\text { component } \alpha \text {-chain }\end{array}$ & MM & 43295/8.35 & 6 & 6 & 15 & 110 & $34 / C$ \\
\hline P05091 & Aldehyde dehydrogenase & $\mathrm{MM}$ & $56381 / 6.63$ & 10 & 10 & 26 & 179 & $31 / C$ \\
\hline O75439 & $\begin{array}{l}\text { Peptidase (mitochondrial } \\
\text { processing) beta }\end{array}$ & MM & $54167 / 6.15$ & 15 & 8 & 21 & 107 & $32 / C$ \\
\hline P09622 & $\begin{array}{l}\text { Dihydrolipoamide } \\
\text { dehydrogenase }\end{array}$ & MM & $54150 / 7.59$ & 8 & 5 & 12 & 69 & $40 / \mathrm{B}$ \\
\hline
\end{tabular}




\begin{tabular}{|c|c|c|c|c|c|c|c|c|}
\hline P27144 & $\begin{array}{l}\text { Adenylate kinase isoenzyme 4, } \\
\text { mitochondrial }\end{array}$ & MM & $25268 / 8.47$ & 5 & 4 & 21 & 76 & $41 / \mathrm{B}$ \\
\hline P54819 & $\begin{array}{l}\text { Adenylate kinase isoenzyme 2, } \\
\text { mitochondrial }\end{array}$ & IMS & 26346/7.85 & 6 & 5 & 30 & 102 & $42 / \mathrm{B}$ \\
\hline Q9UIJ7 & $\begin{array}{l}\text { Adenylate kinase isoenzyme 3, } \\
\text { mitochondrial }\end{array}$ & MM & 25434/9.16 & 10 & 8 & 30 & 137 & 53/B \\
\hline gi|543064 & $\begin{array}{l}\text { Long chain fatty acid beta } \\
\text { oxidation multienzyme } \\
\text { complex alpha chain precursor, } \\
\text { mitochondrial }\end{array}$ & MM & 82959/9.16 & 25 & 12 & 15 & 123 & 43/B \\
\hline Q16762 & Thiosulfate sulfurtransferase & MM & $33297 / 6.8$ & 6 & 5 & 25 & 93 & $44 / \mathrm{B}$ \\
\hline Q92799 & 3-ketoacyl-CoA thiolase & MM & 24021/8.55 & 6 & 4 & 11 & 58 & $45 / \mathrm{B}$ \\
\hline Q16698 & 4-enoyl-CoA reductase & MM & 36067/9.35 & 11 & 6 & 17 & 88 & $46 / \mathrm{B}$ \\
\hline P00505 & $\begin{array}{l}\text { Aspartate aminotransferase } 2 \\
\text { precursor }\end{array}$ & MM & $47475 / 9.14$ & 8 & 7 & 20 & 117 & $47 / \mathrm{B}$ \\
\hline Q99798 & Aconitase 2 & MM & $85425 / 7.36$ & 11 & 9 & 15 & 124 & $48 / \mathrm{B}$ \\
\hline gi|6912518 & $\begin{array}{l}\text { Acyl-CoA thioesterase 2, } \\
\text { mitochondrial }\end{array}$ & MM & $46354 / 8.09$ & 8 & 6 & 16 & 95 & 49/B \\
\hline
\end{tabular}




\begin{tabular}{|c|c|c|c|c|c|c|c|c|}
\hline 1I10A & $\begin{array}{l}\text { L-lactate dehydrogenase M } \\
\text { chain }\end{array}$ & MM & $36557 / 8.46$ & 10 & 8 & 27 & 132 & $50 / \mathrm{B}$ \\
\hline P42765 & Acetyl-CoA acyltransferase 2 & MM & $41924 / 8.34$ & 13 & 7 & 22 & 94 & $51 / \mathrm{B}$ \\
\hline P40926 & $\begin{array}{l}\text { Malate dehydrogenase, } \\
\text { precursor }\end{array}$ & MM & $35531 / 8.92$ & 15 & 10 & 39 & 136 & $52 / \mathrm{B}$ \\
\hline Q9ULV4 & Coronin 3 & $\mathrm{U}$ & $53249 / 6.65$ & 12 & 6 & 14 & 77 & 33/A \\
\hline P09211 & Glutathione S-transferase P & $\mathrm{M}$ and $\mathrm{C}$ & $25365 / 8.53$ & 22 & 6 & 43 & 84 & $30 / \mathrm{C}$ \\
\hline Q96EZ9 & $\begin{array}{l}\text { Delta 3,4-delta 2,4 dienoyl- } \\
\text { CoA isomerase }\end{array}$ & $\mathrm{M}$ and $\mathrm{P}$ & $35816 / 8.16$ & 21 & 7 & 24 & 83 & $17 / \mathrm{A}$ \\
\hline P35232 & Prohibitin & $\mathrm{M}$ and $\mathrm{C}$ & $29804 / 5.57$ & 23 & 12 & 56 & 165 & $35 / \mathrm{A}$ \\
\hline
\end{tabular}

${ }^{a}$ Accession numbers in SWISS-PROT or NCBI databases are given. ${ }^{b}$ Subcellular location as annotated in the SWISS-PROT database is indicated: M, mitochondria; MIM, mitochondrial inner membrane; MM, mitochondrial matrix; OMM, outer mitochondrial membrane; C, cytosol; P, peroxisome; U, ubiquitous. ${ }^{\mathrm{T}}$ Theoretical molecular mass and $\mathrm{p} I$ values were determined using SWISS-PROT database. ${ }^{d}$ Numbers refer to spot numbers as given in Supplementary Figure. 
Supplementary table B. Other proteins also present in the sample preparation.

\begin{tabular}{|c|c|c|c|c|c|c|c|c|}
\hline $\begin{array}{l}\text { Accession } \\
\text { number }^{a}\end{array}$ & Protein name & Location $^{b}$ & $\mathbf{M r} / \mathbf{p} I^{c}$ & $\begin{array}{c}\text { Number of } \\
\text { masses searched }\end{array}$ & $\begin{array}{c}\text { Number of } \\
\text { masses matched }\end{array}$ & $\begin{array}{c}\text { Sequence } \\
\text { coverage \% }\end{array}$ & $\begin{array}{c}\text { MASCOT } \\
\text { score }\end{array}$ & $\begin{array}{l}\text { No. }{ }^{d} / \\
\text { Figure }\end{array}$ \\
\hline P04792 & Hsp27 & $\mathrm{C}$ & $22327 / 7.83$ & 14 & 7 & 33 & 115 & $54 / \mathrm{A}$ \\
\hline Р30041 & Antioxidant protein 2 & $\mathrm{C}$ and $\mathrm{L}$ & 24903/6.03 & 19 & 7 & 25 & 96 & $55 / \mathrm{A}$ \\
\hline Q13162 & Peroxiredoxin 4 & $\mathrm{C}$ & 30539/5.86 & 10 & 5 & 32 & 85 & 56/A \\
\hline P35579 & Myosin heavy chain & $\mathrm{C}$ & $226532 / 5.50$ & 19 & 14 & 19 & 85 & 57/A \\
\hline P08670 & Vimentin & $\mathrm{C}$ & $53554 / 5.00$ & 23 & 14 & 39 & 256 & 58/A \\
\hline P02570 & Actin, cytoplasmic 1 & $\mathrm{C}$ & $41736 / 5.29$ & 23 & 9 & 26 & 113 & $59 / \mathrm{C}$ \\
\hline P05209 & Alpha tubulin 1 & $\mathrm{C}$ & $50151 / 4.94$ & 4 & 4 & 14 & 84 & $60 / \mathrm{C}$ \\
\hline P05217 & Tubulin beta-2 chain & $\mathrm{C}$ & $49831 / 4.79$ & 20 & 9 & 21 & 111 & $61 / \mathrm{C}$ \\
\hline P18206 & Vinculin & $\mathrm{C}$ & $123668 / 5.51$ & 14 & 9 & 8 & 78 & $62 / \mathrm{C}$ \\
\hline O43707 & Alpha-actinin 4 & $\mathrm{~N}$ and $\mathrm{C}$ & $104854 / 5.27$ & 25 & 12 & 13 & 116 & $63 / \mathrm{A}$ \\
\hline Q14697 & Glucosidase II precursor & ER & $106900 / 5.71$ & 24 & 21 & 20 & 275 & 64/A \\
\hline A59144 & $\begin{array}{l}\text { Procollagen-lysine-5- } \\
\text { dioxygenase } 2 \text { precursor }\end{array}$ & ER & $84663 / 6.15$ & 9 & 8 & 12 & 77 & $65 / \mathrm{C}$ \\
\hline Q9UBS4 & $\begin{array}{l}\text { ER-associated hsp40 co- } \\
\text { chaperone }\end{array}$ & ER & $40513 / 5.81$ & 12 & 6 & 14 & 83 & $66 / \mathrm{C}$ \\
\hline
\end{tabular}




\begin{tabular}{|c|c|c|c|c|c|c|c|c|}
\hline P30101 & $\begin{array}{l}\text { Protein disulfide-isomerase } \\
\text { ER60 precursor }\end{array}$ & ER & $56782 / 5.98$ & 7 & 5 & 9 & 75 & $67 / \mathrm{C}$ \\
\hline T09549 & $\begin{array}{l}\text { Endoplasmic-reticulum- } \\
\text { lumenal protein } 28\end{array}$ & ER & 28993/6.77 & 18 & 8 & 32 & 114 & $68 / \mathrm{C}$ \\
\hline P04083 & Annexin I & $\mathrm{CM}$ & $38583 / 6.64$ & 9 & 8 & 32 & 148 & $69 / \mathrm{C}$ \\
\hline P08758 & Annexin V & $\mathrm{CM}$ & $35805 / 4.94$ & 20 & 12 & 42 & 143 & $70 / \mathrm{A}$ \\
\hline P08133 & Annexin VI & $\mathrm{CM}$ & $75742 / 5.42$ & 18 & 18 & 28 & 290 & $71 / \mathrm{C}$ \\
\hline Q9NZM1 & Myoferlin & $\mathrm{CM}$ & $234708 / 5.84$ & 16 & 11 & 6 & 95 & $72 / \mathrm{C}$ \\
\hline P26038 & Moesin & $\mathrm{CM}$ & 67688/6.00 & 23 & 14 & 20 & 172 & $73 / \mathrm{C}$ \\
\hline gi|4557305 & Aldolase A & $\mathrm{C}$ & $39420 / 8.30$ & 11 & 7 & 28 & 111 & $74 / \mathrm{B}$ \\
\hline gi|4505763 & $\begin{array}{l}\text { Phosphoglycerate } \\
\text { kinase } 1\end{array}$ & $\mathrm{C}$ & $44614 / 8.30$ & 13 & 9 & 29 & 142 & $75 / \mathrm{B}$ \\
\hline gi|999892 & Triosephosphate isomerase & $\mathrm{C}$ & $26538 / 6.51$ & 11 & 8 & 49 & 146 & $76 / \mathrm{B}$ \\
\hline gi|4505753 & $\begin{array}{l}\text { Phosphoglycerate } \\
\text { mutase } 1\end{array}$ & $\mathrm{C}$ & $28803 / 6.67$ & 10 & 10 & 45 & 210 & $77 / \mathrm{B}$ \\
\hline gi|123576 & 47kDa heat shock protein & ER & $46267 / 8.27$ & 7 & 5 & 14 & 77 & $78 / \mathrm{B}$ \\
\hline gi|4503571 & Enolase 1 & C & $47168 / 7.01$ & 11 & 8 & 23 & 123 & $79 / \mathrm{B}$ \\
\hline
\end{tabular}




\begin{tabular}{|c|c|c|c|c|c|c|c|c|}
\hline gi|179950 & Catalase & $\mathrm{C}$ & $51353 / 7.84$ & 10 & 9 & 25 & 151 & $80 / \mathrm{B}$ \\
\hline gi|20178296 & Pyruvate kinase M1 & $\mathrm{C}$ & $57936 / 7.96$ & 10 & 7 & 16 & 128 & $81 / \mathrm{B}$ \\
\hline gi|478822 & Pyruvate kinase M2 & $\mathrm{C}$ & $57877 / 7.58$ & 8 & 5 & 13 & 93 & $82 / \mathrm{B}$ \\
\hline gi|31455214 & MPHOSPH1 protein & $\mathrm{N}$ & $72517 / 5.39$ & 8 & 5 & 11 & 63 & 83/B \\
\hline gi|31874555 & Hypothetical protein & & $64802 / 6.85$ & 12 & 8 & 19 & 110 & 84/B \\
\hline gi|41472053 & Unknown & & 33228/8.54 & 24 & 8 & 30 & 130 & $85 / \mathrm{B}$ \\
\hline gi|13375874 & Hypothetical protein FLJ23393 & & $43114 / 8.76$ & 6 & 4 & 11 & 63 & $86 / \mathrm{B}$ \\
\hline gi|3859998 & Unknown & & $36628 / 5.85$ & 4 & 3 & 11 & 64 & $87 / \mathrm{B}$ \\
\hline gi|9506611 & Hypothetical protein FLJ10613 & & $66216 / 8.68$ & 11 & 5 & 21 & 81 & 88/B \\
\hline gi|7705302 & Hypothetical protein BM-005 & & 75284/8.96 & 20 & 8 & 16 & 109 & 89/B \\
\hline gi|22760314 & Unnamed product & & $47046 / 6.66$ & 10 & 8 & 22 & 130 & $90 / \mathrm{B}$ \\
\hline Q9NVA2 & Hypothetical protein FLJ10849 & & 49398/6.36 & 22 & 11 & 25 & 142 & $91 / \mathrm{A}$ \\
\hline CAD23331 & Sequence 1 from patent & & 101745/6.13 & 15 & 13 & 16 & 156 & $92 / \mathrm{A}$ \\
\hline & WO0200863 & & & & & & & \\
\hline Q96CB6 & Hypothetical $61.2 \mathrm{kDa}$ protein & & $61161 / 6.00$ & 19 & 11 & 20 & 141 & 93/A \\
\hline O94905 & Hypothetical $37.8 \mathrm{kDa}$ protein & & $37839 / 5.47$ & 20 & 10 & 31 & 140 & $94 / \mathrm{C}$ \\
\hline Q96A39 & Hypothetical 41.9 kDa protein & & $41920 / 5.88$ & 12 & 7 & 23 & 78 & $95 / \mathrm{C}$ \\
\hline Q9BUM6 & Hypothetical $47.5 \mathrm{kDa}$ protein & & $47450 / 5.22$ & 12 & 7 & 14 & 97 & $96 / \mathrm{C}$ \\
\hline
\end{tabular}




\begin{tabular}{lllllccc}
\hline T23493 & Hypothetical protein K08F8.5b & $34902 / 6.66$ & 16 & 9 & 8 & 78 & $97 / \mathrm{C}$ \\
Q9BU08 & Unknown & $59468 / 5.45$ & 12 & 7 & 14 & 93 & $98 / \mathrm{C}$ \\
gi|4406579 & Unknown & $48747 / 7.11$ & 7 & 6 & 19 & 102 & $99 / \mathrm{B}$ \\
gi|33870117 & Unknown & $62046 / 8.99$ & 7 & 6 & 12 & 92 & $100 / \mathrm{B}$
\end{tabular}

${ }^{a}$ Accession numbers in SWISS-PROT or NCBI databases are given. ${ }^{b}$ Subcellular location as annotated in the SWISS-PROT database is indicated: C, cytosol; N, nucleus; ER, endoplasmic reticulum; CM, cell membrane; L, lysosome. ${ }^{\mathrm{C}}$ Theoretical molecular mass and $\mathrm{pI}$ values were determined using SWISS-PROT database. ${ }^{d}$ Numbers refer to spot numbers as given in Supplementary Figure. 
Supplementary Figure

A

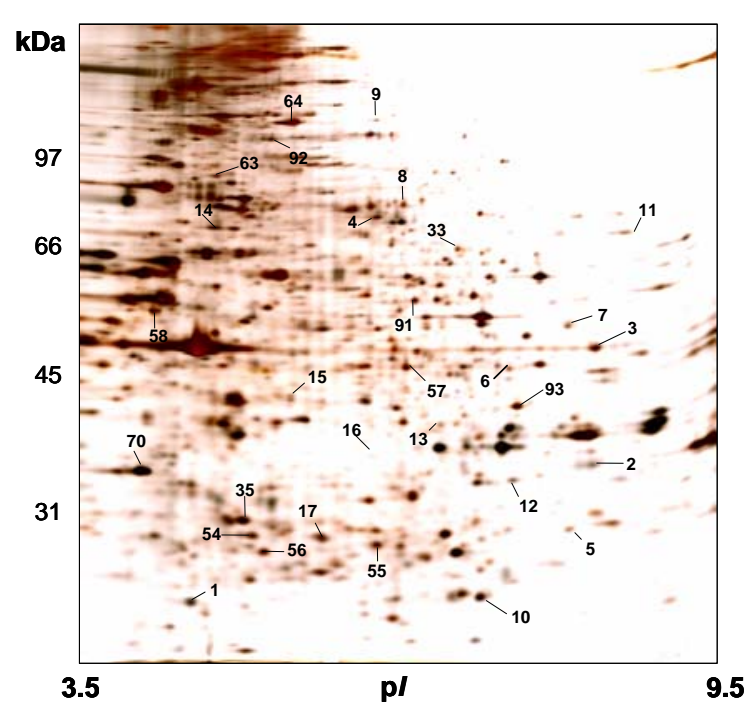

B

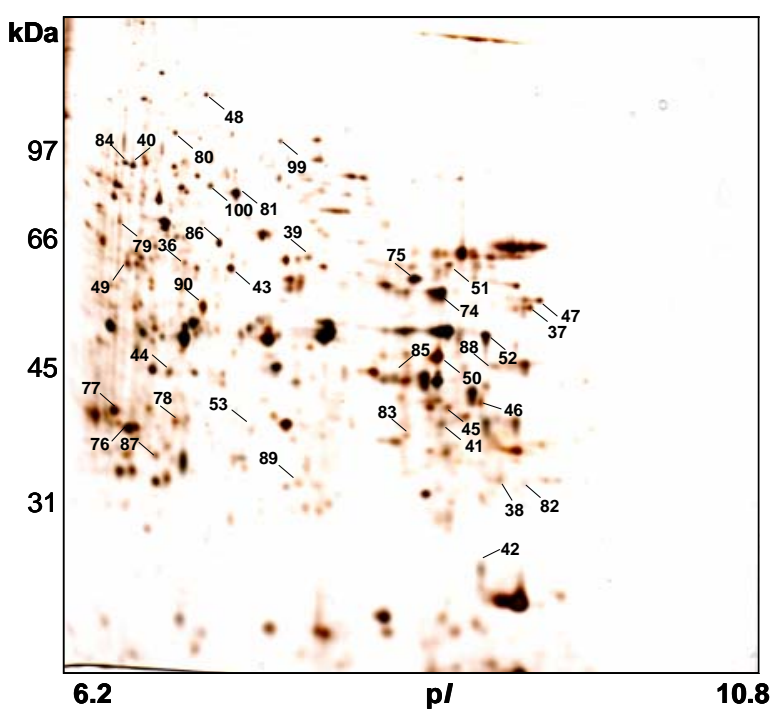

C

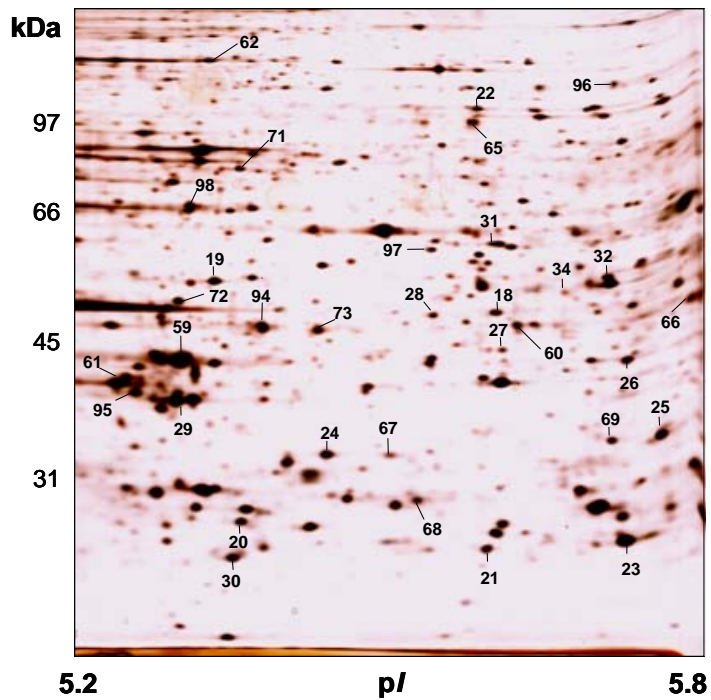

